\title{
APORTACIONES AL DISEÑO DE FORMACIÓN DE EMPRENDEDORES DESDE LA MEDICIÓN DE ACTITUDES DEL ESTUDIANTE UNIVERSITARIO. CASO UAEMEX - UPTC (2016)
}

G. Gonzalez-Garcia ${ }^{1}$, C. E. Estrada-Gutierrez ${ }^{2}$, E. Leite ${ }^{3}$, J. Alvarez-Botello ${ }^{4}$

${ }^{1,2,4}$ Universidad Autónoma del Estado de México, ${ }^{3}$ Universidade de Pernambuco guadalupe_ggg@hotmail.com

Submetido 24/01/2017 - Aceito 07/05/2017

DOI: $10.15628 /$ holos. 2017.5577

\section{RESUMEN}

La cultura formadora de emprendedores capaces de ofrecer servicios de manera independiente, crear empresas o innovar en las existentes, es sin duda una de las tareas universitarias. El objetivo de esta investigación fue sentar bases para el diseño de formación emprendedora a partir de elaborar un perfil. Se describen los valores para características personales de estudiantes de licenciatura en Administración en dos universidades públicas en diferentes países. Los resultados indican que existe una estrecha relación entre algunas dimensiones. La comparación posibilita identificar áreas de oportunidad para ser aprovechadas por los estudiantes mexicanos y colombianos. Las conclusiones orientarán la cultura emprendedora de instituciones educativas.

PALABRAS-CLAVE: emprendedores, universidades públicas, formación universitaria, emprendimiento

\section{CONTRIBUTIONS TO THE DESIGN OF TRAINING OF ENTREPRENEURS FROM THE MEASUREMENT OF UNIVERSITY STUDENT ATTITUDES. CASE UAEMEX - UPTC 2016}

\begin{abstract}
The culture that forms entrepreneurs able to offer services independently, to create companies or to innovate in existing ones is, without a doubt, one of the university tasks. The objective of this research was to lay the foundations for the design of entrepreneurial training from the elaboration of a profile. The values for personal characteristics of undergraduate students in
\end{abstract}

Administration, in two public universities in different countries are described. The results indicate that there is a close relationship among some dimensions. The comparison makes it possible to identify areas of opportunity to be used by Mexican and Colombian students. The conclusions will guide the entrepreneurial culture of educational institutions.

KEYWORDS: Entrepreneurs, public universities, university education, entrepreneurship 


\section{Introducción}

El impulso a emprendedores con proyectos innovadores se ha convertido en un eje de acción en muchas universidades. Forjar profesionales en distintas áreas del conocimiento inherentemente marca la diferencia en un contexto de desarrollo humano con hábitos, actitudes y valores determinantes para fomentar el emprendimiento. Al tomar conciencia de lo que implica, se promueven planes de estudio con una visión para el mejor desempeño en el contexto laboral; destacando entre otros asuntos la cultura empresarial y emprendedora así que el egresado sea capaz de ofrecer servicios de forma independiente, crear empresas o innovar en las existentes.

Emprender exige espíritu y una mentalidad que hacen que los individuos no esperen, sino que actúen para enfrentarse a un mercado que no perdona errores y que cada día se vuelve más riguroso. Hoy, se exige una mayor preparación y esmero en la administración de los negocios. La diferencia entre la educación profesional que fomenta una cultura emprendedora y la que no, tal y como mencionan Bygrave y Minniti (2000), es que recure a modelos de emprendedor en el entorno del estudiante para influir sobre su decisión y se asocian con la probabilidad de concretar con éxito sus proyectos. También impulsa profesionistas emprendedores debido a que las características del perfil congruente se refuerzan a través de la interiorización de los conocimientos y destrezas.

El concepto de cultura emprendedora definido por Gibb (1999: 27-38) como el "conjunto de valores, creencias y aptitudes comúnmente compartidas en una sociedad" sostiene la idea de que es deseable un modo de vida emprendedor apoyando continuamente la búsqueda de un comportamiento emprendedor efectivo por parte de los individuos o grupos. Esta investigación plantea la necesidad de conocer las características empresariales a partir de los cuales se pueden diseñar programas de estudio innovadores que permitan a los alumnos desarrollar y mejorar habilidades y conocimientos para el emprendimiento.

\section{Revisión bibliográfica}

\section{Emprendimiento}

El término emprender desde su origen en el siglo XVIII ya utilizado por el economista irlandés Richard Cantillon quien definió al emprendedor como "agente que compra medios de producción a precios determinados con el fin de combinarlos dentro de un nuevo producto", más adelante el economista francés Jean-Baptiste Say menciona que "es alguien que une a otras personas con el fin de crear un organismo único de producción" (Schumpeter, 1951). Son muchos los estudiosos que han seguido el concepto del emprendimiento hasta nuestros días, como Howard Rasheed (citado por Duarte et al, 2009) que plantea que "la educación en entrepreneurship puede afectar los atributos que tengan los individuos y puede forjar actitudes emprendedoras en ellos".

Formichella (2002) cita entre otros a Stevenson que apunta: emprender "es perseguir la oportunidad más allá de los recursos que se controlen en la actualidad"; a Kundell: "la gestión del cambio radical y discontinuo, o renovación estratégica, sin importar si esta renovación estratégica ocurre adentro o afuera de organizaciones existentes, y sin importar si esta renovación da lugar, o no, a la creación de una nueva entidad de negocio"; a Drucker discurriendo que es "el medio con el cual explotar el cambio como una oportunidad para un negocio diferente" para concluir que tener éxito al realizar una actividad es una cuestión de suerte. 
Es importante tener claro cuáles son las verdaderas causas que inducen que un emprendimiento resulte favorable, siendo imprescindible destacar que, en realidad, el éxito se relaciona estrechamente con la actitud. Son principalmente dos los pensamientos básicos acerca del emprendedor delimitados por Rodríguez (2009) en su artículo de construcción ecléctica y holística: el reconocido por sus características personales, que arriesga en una hazaña y el reconocido por sus funciones, que planea estrategias y metas. Ahora bien, según el contexto en que se utilice, el emprendedor en los negocios es un empresario o propietario de una empresa con fines de lucro, mientras que en el entorno académico más bien describe un perfil o un conjunto de características que hacen actuar a los individuos de una manera determinada y demostrar ciertas competencias para definir y alcanzar objetivos (Finley, 1990 y Ronstad, 1985 citados por Alcaraz, 2011).

Emprendimiento o emprendedurismo, palabras utilizadas casi de igual manera como traducción de entrepreneurship, es la forma de abordar el espíritu emprendedor definido en el Diccionario de la Real Academia como la cualidad de emprendedor. El Global Entrepreneurship Monitor (GEM) lo define como "cualquier intento llevado a cabo por los individuos para empezar una nueva empresa, incluyendo el de volverse auto-empleado". Especialmente es decisivo para el desarrollo de las economías emergentes como motores de inversión, empleo e innovación. El emprendimiento no se crea de forma espontánea ya que depende de un ambiente propicio con métodos funcionales (Brenes y Haar, 2016:10).

Los jóvenes tienen derechos para desarrollar todo su potencial en el ámbito laboral (Naciones Unidas, 2004); la Organización Internacional del Trabajo (OIT) establece que la formación profesional consiste en "actividades que tienden a proporcionar la capacidad práctica, el saber y las actitudes necesarias para el trabajo en una ocupación o grupo de ocupaciones en cualquier rama de la actividad económica", así las funciones sustantivas de las universidades están encaminadas a proveer a los estudiantes con los contenidos teóricos y desarrollo de competencias para la vida profesional, y que hoy en día requiere el emprendedor ya sea de carácter interno o externo de las organizaciones.

Por último, aunque es evidente que sin ser exhaustivo al respecto, se retoma que la UNESCO (por sus siglas en inglés de United Nations Educational, Scientific and Cultural Organization) en su declaración de Incheon 2030 (Corea) sobre la educación, reconoce la importancia que desempeña la educación como motor principal del desarrollo, así como clave para lograr el pleno empleo y la erradicación de la pobreza, para lo cual se requiere la equidad, la inclusión, la calidad y los resultados del aprendizaje, dentro de un enfoque del aprendizaje a lo largo de toda la vida. Ante esto, promover y generar emprendimiento en los estudiantes de educación superior se vuelve una competencia necesaria de los programas de estudio.

\section{Emprendimiento en México y Colombia}

En México convergen programas gubernamentales orientados al apoyo a emprendedores, ejemplo de ello es el Instituto Nacional del Emprendedor INADEM creado en 2013 y que tiene que tiene por objeto instrumentar, ejecutar y coordinar la política nacional de apoyo incluyente a emprendedores y a las micro, pequeñas y medianas empresas, impulsando su innovación, competitividad y proyección en los mercados nacional e internacional para aumentar su contribución al desarrollo económico y bienestar social, así como coadyuvar al desarrollo de políticas que fomenten la cultura y productividad empresarial ${ }^{1}$.

Colombia cuenta con iniciativas de Gobierno Nacional, como: SENA (Servicio Nacional de Aprendizaje) a través del Fondo Emprender, cuyo propósito es ofrecer capital semilla para

\footnotetext{
${ }^{1}$ Para ver más seguir la liga https://www.inadem.gob.mx/index.php/conoce-inadem
} 
desarrollar planes de negocio en diversos campos; INNpulsa Colombia ${ }^{2}$ que fomenta la mentalidad y la cultura emprendedora, cerrar las brechas de mercado e impulsar las empresas; Apps.co, enfocada en el emprendimiento TIC. Básicamente el Fondo Emprender busca incentivar y facilitar la generación y creación de empresas, contribuyendo al desarrollo humano e inserción de emprendedores en el sector productivo. Se rige por el derecho privado y su presupuesto está conformado por el $80 \%$ de la monetización de la cuota de aprendizaje, así como por los aportes del presupuesto general de la nación, recursos financieros de: organismos de cooperación nacional e internacional, la banca multilateral, fondos de pensiones y cesantías y fondos de inversión públicos y privados.

Es significativo para nuestra investigación tener un referencial actualizado sobre el emprendimiento y el desarrollo, por lo que se usó el índice 2016 del Global Entrepreneurship Monitor $^{3}$, donde México se encuentra en el lugar número 87 y Colombia en el lugar 42 de un total de 132 países; llama la atención que ambos países aparecen en el biggest declines (países con las mayores caídas) in the GEI Score 2016 tal y como se aprecia en la Tabla 1.

\begin{tabular}{|c|c|c|c|c|}
\hline Country & Score 2016 & Score 2015 & $\begin{array}{c}\text { Difference in } \\
\text { score }\end{array}$ & $\begin{array}{c}\text { Difference in } \\
\text { ranking }\end{array}$ \\
\hline United Kingdom & 67.7 & 72.7 & -5.01 & 5 \\
\hline Czech Republic & 44.2 & 48.9 & -4.65 & 9 \\
\hline Norway & 61.1 & 65.6 & -4.52 & 5 \\
\hline Angola & 18.6 & 22.7 & -4.11 & 1 \\
\hline El Salvador & 25.6 & 29.6 & -4.05 & 15 \\
\hline Finland & 61.8 & 65.7 & -3.92 & 4 \\
\hline Belgium & 62.1 & 65.5 & -3.43 & 1 \\
\hline Colombia & 44.8 & 47.9 & -3.19 & 7 \\
\hline Mexico & 27.6 & 30.7 & -3.03 & 12 \\
\hline Malaysia & 37.0 & 40.0 & -2.97 & 3 \\
\hline
\end{tabular}

Tabla 1. Países con mayores caídas. Fuente: Global Entrepreneurship and Development Institute. https://thegedi.org/global-entrepreneurship-and-development-index/recuperado el 30 de marzo de 2016

\section{Características empresariales personales}

El emprendimiento es una disciplina susceptible de ser enseñada y aprendida, (Kuratko, 2003:5, citado por Paez, 2011) y sus habilidades pueden ser fomentadas como Campos et al (2014) discurren que Aronsson (2004) y Kirby (2004) encontraron evidencia empírica que demuestra que la adquisición de conocimientos sobre creación de empresas puede ser un factor que influya en el desarrollo de habilidades emprendedoras, ya que Aronsson por una parte señala que el espíritu emprendedor puede ser alentado a través de la enseñanza y por otra, Kirby afirma que las capacidades empresariales no sólo son innatas sino que también pueden ser adquiridas a través del aprendizaje. Spinelli y Adams (2012) plantean el emprendimiento como el nuevo paradigma de la Administración. Se aprecia que recientemente las empresas han estrechado el lazo exitoso que une emprender e innovar, asegura Prieto (2014).

\footnotetext{
2 Para ver más seguir la liga http://www.fondoemprender.com/SitePages/Home.aspx

3 Global Entrepreneurship Monitor constituido desde 2008 por investigadores de: Alemania, Angola, Argentina, Bélgica, Bolivia, Bosnia y Herzegovina, Brasil, Chile, Colombia, Corea, Croacia, Dinamarca, Ecuador, Egipto, Eslovenia, España, Estados Unidos, Finlandia, Francia, Grecia, Holanda, Hungría, India, Irán, Irlanda, Islandia, Israel, Italia, Jamaica, Japón, Letonia, Macedonia, México, Noruega, Perú, Reino Unido, República Dominicana, Rumania, Rusia, Serbia, Sudáfrica, Turquía y Uruguay. Para ver más, seguir la liga www.gemconsortium.org.
} 


\section{Metodología}

Esta investigación de carácter no experimental ha sido dirigida a estudiantes de licenciatura en Administración de la Universidad Autónoma del Estado de México UAEMEX (120 alumnos) y de la Universidad Pedagógica y Tecnológica de Colombia UPTC (100 alumnos), sin considerar ningún parámetro específico de edad, sexo o semestre. El tamaño de la muestra indica margen de error de $8 \%$ y un nivel de confianza de $95 \%$, elegido así ya que el perfil de egreso entre otros aspectos incluye el emprendimiento. Es de tipo descriptivo ya que busca establecer el nivel de las Características Empresariales Personales, identificando conductas y actitudes que llevan a construir comportamientos concretos, para descubrir y determinar las relaciones entre las diversas variables del estudio; es transversal con datos del año 2016. Se aplica un cuestionario a estudiantes de universidades públicas en dos países con la intención también de identificar similitudes y diferencias. La pregunta que se busca clarificar es ¿cómo sería diseñar una formación para emprendedores a partir de un perfil CEP?

\section{Resultados y discusión}

En el proceso de cambio para incentivar los comportamientos emprendedores de los estudiantes de Administración en la universidad es importante primero determinar las características personales con que cuentan. La investigación acerca de ellas considera tres aspectos (Uriarte y González, 2007; citado en Paez, 2011): 1) La existencia de característica de índoles psicológicas, sociales, hereditarias, ambientales, educacionales o experimentales que diferencian al emprendedor con éxito de otros grupos humanos. 2) Que ellas existan previamente al comportamiento emprendedor. 3) Determinar la posibilidad de evaluarlas y anticiparlas al proceso de la creación de empresas.

Es primordial fomentar una cultura emprendedora conforme con Vargas (2007) como marco de referencia de valores que moldeen las actividades productivas de sus principales actores: empresarios y administradores. De ahí que, como menciona Gilder (1984) es importante determinar las características empresariales, para comprender cómo los empresarios son personas capaces de aprender pero que también persiguen sus objetivos a pesar de fracasos y frustraciones, $y$, finalmente, obtienen el éxito a través de romper viejos patrones y crear su propio nuevo orden.

Cano et al (2003) destacan en su análisis de actitudes emprendedoras de los estudiantes universitarios, que en lo relativo al fomento de la cultura emprendedora el interés se ha traducido en diversas acciones como la inclusión de asignaturas específicas sobre creación de empresas en algunos planes de estudio. Veciana y Urbano (2000) recomiendan unidades de apoyo al autoempleo y el desarrollo de acciones concretas como concursos de ideas empresariales o incubadoras de empresas. Concluye Paez (2011) que describir el perfil emprendedor requiere también introducirse en la intención y así determinar quiénes de los que cuentan con un potencial terminan convirtiéndose en reales emprendedores y empresarios.

Para la recolección de datos se tomó como referencia el cuestionario de Management Systems International a fin de conocer el nivel de las características empresariales personales de los alumnos en estudio ya que revisa las requeridas para conformar una empresa y se puede elaborar el propio perfil que marca tendencias en una u otra dirección. No tiene necesariamente un valor en términos absolutos y su efectividad e impacto entre los participantes es precisamente su carácter neutral y despersonalizado. En las 55 preguntas se evalúan diez características respondidas bajo el sistema Likert, agrupadas en las siguientes dimensiones: 
Iniciativa. Persistencia. Cumplimiento. Calidad. Arriesgue. Metas. Información. Planificación. Redes. Autoconfianza.

Ejemplo:

"CARACTERISTICAS EMPRESARIALES PERSONALES CER"

Facultad de Administración de la Universidad Pedagógica y Tecnológica de Colombia. Facultad de Contaduría y Administración de la Universidad Autónoma del Estado de México.

El propósito de este cuestionario es revisar en forma sintética las CER requeridas para conformar una empresa que permitan realimentar nuestros planes de estudio, en función a las nuevas exigencias. Las respuestas serán utilizadas solamente para efectos académicos. Agradezco por su colaboración para responder este documento. Dra. en A. Guadalupe González García.

\section{Datos Generales}

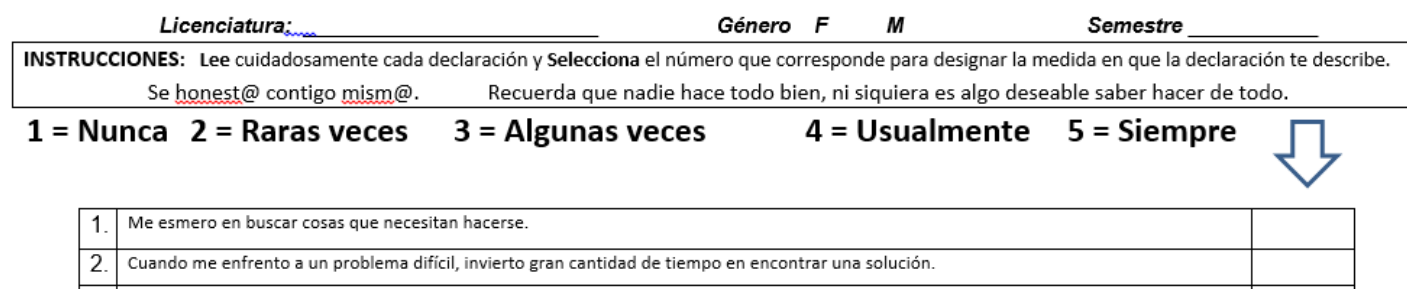

Resultados

Las respuestas se realizaron en presencia de los encuestadores obteniéndose una Alfa de Cronbach .864 lo que da una confiabilidad bastante buena en los resultados obtenidos. En la Gráfica 1. se muestran los resultados correspondientes a Colombia, en la Gráfica 2. se observan los valores para México, destacando que:

- Los valores más altos en Colombia se encontraron en Metas, Persistencia y Cumplimiento.

- Los valores más altos en México fueron Metas, Autoconfianza y Cumplimiento.

- Los valores anteriores indican que los estudiantes de Administración de ambos países son muy parecidos, a excepción de la Autoconfianza, que como se puede observar es mayor en el caso de los mexicanos y la persistencia en el caso de los colombianos.

- Los valores mínimos para el caso de Colombia fueron Planificación, Redes e Información.

- Los valores mínimos para el caso de México fueron Calidad, Arriesgue y Redes.

Al compararlos en la Gráfica 3. se puede observar que Colombia tiene cierta ventaja en Calidad y Arriesgue, pero se encuentra cierta desventaja en Metas, Planificación y Autoconfianza.

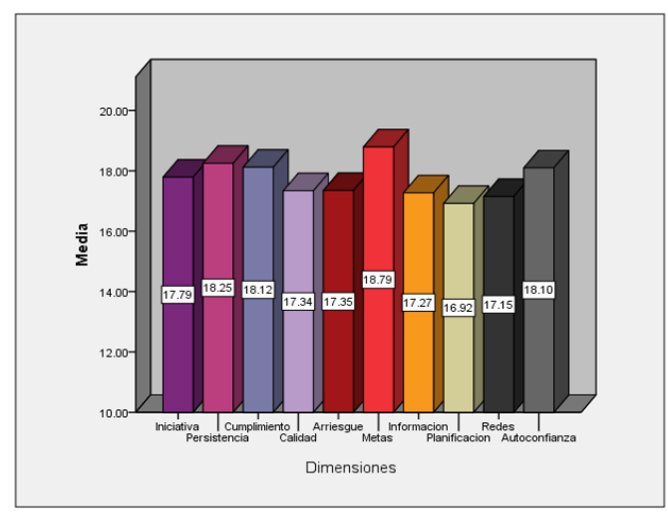

Gráfica 1. Colombia

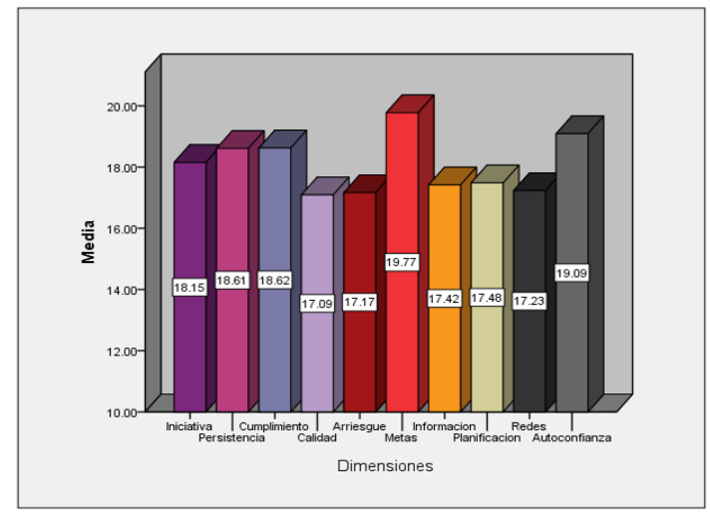

Gráfica 2. México 


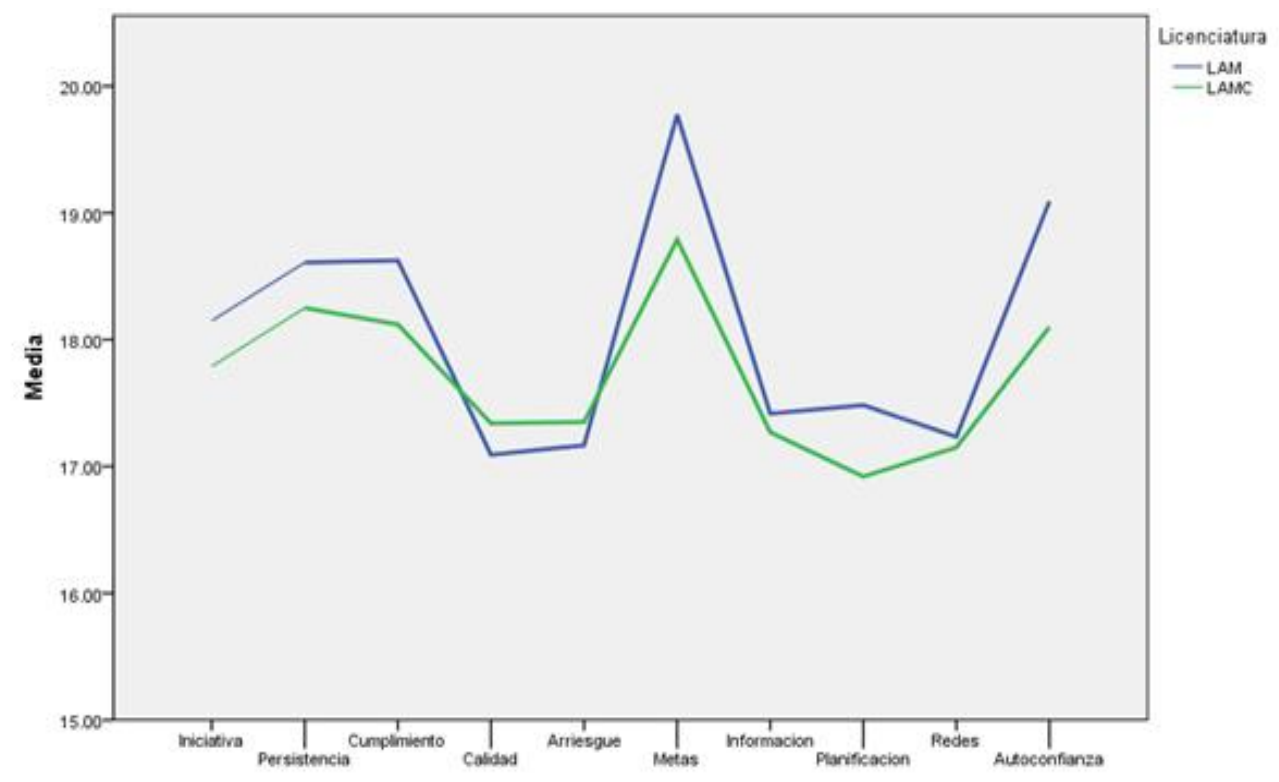

Gráfica 3. Comparativo México - Colombia

Se usó el coeficiente de correlación de Pearson para identificar algunas relaciones entre las características correspondientes, que sobresalieron del instrumento encontrando que, a Mayor Iniciativa, Mayor Persistencia y a Mayor Planificación, Mayor Información, esto al obtener valores mayores a .4 en la correlación y menores a .05 en el Sig. (Bilateral) entre las variables correspondientes. En la Tabla 2 se muestra los resultados a detalle del total de la muestra que es de 220 estudiantes.

\begin{tabular}{|c|c|c|c|c|c|}
\hline \multicolumn{2}{|r|}{ Correlaciones } & Iniciativa & Persistencia & Planificación & Información \\
\hline \multirow[t]{3}{*}{ Iniciativa } & Correlación de Pearson & 1 & $.438^{* 8}$ & $.261^{88}$ & $.375^{* *}$ \\
\hline & Sig. (bilateral) & & .000 & .000 & .000 \\
\hline & $\mathrm{N}$ & 220 & 220 & 220 & 220 \\
\hline \multirow[t]{3}{*}{ Persistencia } & Correlación de Pearson & $.438^{4 *}$ & 1 & $.324^{* *}$ & $.379^{* *}$ \\
\hline & Sig. (bilateral) & .000 & & .000 & .000 \\
\hline & $\mathrm{N}$ & 220 & 220 & 220 & 220 \\
\hline \multirow[t]{3}{*}{ Planificación } & Correlación de Pearson & $.261^{* *}$ & $.324^{* *}$ & 1 & $.421^{* *}$ \\
\hline & Sig. (bilateral) & .000 & .000 & & .000 \\
\hline & $\mathrm{N}$ & 220 & 220 & 220 & 220 \\
\hline \multirow[t]{3}{*}{ Información } & Correlación de Pearson & $.375^{\circ *}$ & $.379^{4 *}$ & $.421^{4 *}$ & 1 \\
\hline & Sig. (bilateral) & .000 & .000 & .000 & \\
\hline & $\mathrm{N}$ & 220 & 220 & 220 & 220 \\
\hline
\end{tabular}

Tabla 2. Correlaciones de las Características Iniciativa, Persistencia, Planificación e Información 
De estas características que se encuentran en las relaciones, se aplica el estadístico T de Student para muestras independientes, ya que son dos grupos de diferentes países, así que la primera pregunta que nos hacemos es si la Iniciativa es diferente en México que en Colombia, demostrada a través de este estadístico y dio como resultado como se observa en la Tabla 3 . que la media de Iniciativa es de 18.15 para México y 17.79 para Colombia; deseamos saber si realmente son diferencias significativas, así que al aplicar el estadístico tenemos que el $\mathrm{P}$ valor 0 el Sig (bilateral) es de .262 como se aprecia en la Tabla 4, esto es mayor al 0.05, lo que significa que no existen diferencias significativas entre ambos países en la característica de Iniciativa.

Estadísticos de grupo

\begin{tabular}{|r|r|r|r|r|r|}
\hline & Licenciztura & \multicolumn{1}{|c|}{$\mathrm{N}$} & \multicolumn{1}{c|}{ Media } & \multicolumn{1}{c|}{ Desviación tip. } & Error tin, de la media \\
\hline \multirow{2}{*}{ Iniciativa } & LAM & 120 & 18.1500 & 2.38218 & .21746 \\
& LAMC & 100 & 17.7900 & 2.34109 & .23411 \\
\hline
\end{tabular}

Tabla 3. Medias

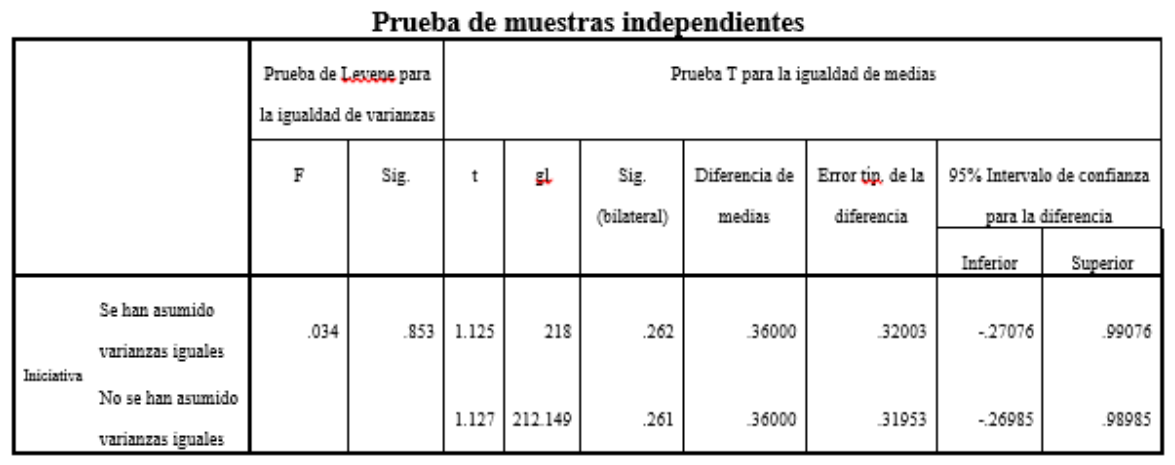

Tabla 4. T de Student

El mismo resultado se observó para las características de Persistencia, Planificación e Información, por lo que podemos concluir que no existen diferencias significativas en las medias de ambos países y por tanto las correlaciones obtenidas son válidas en ambos casos.

En la Tabla 5. se aprecia que para Metas y Autoconfianza sí existen diferencias significativas entre ambos países ya que el Sig (Bilateral) o $\mathrm{P}$ valor se encuentra por debajo del 0.05 y esto es algo que Colombia debería trabajar en sus emprendedores.

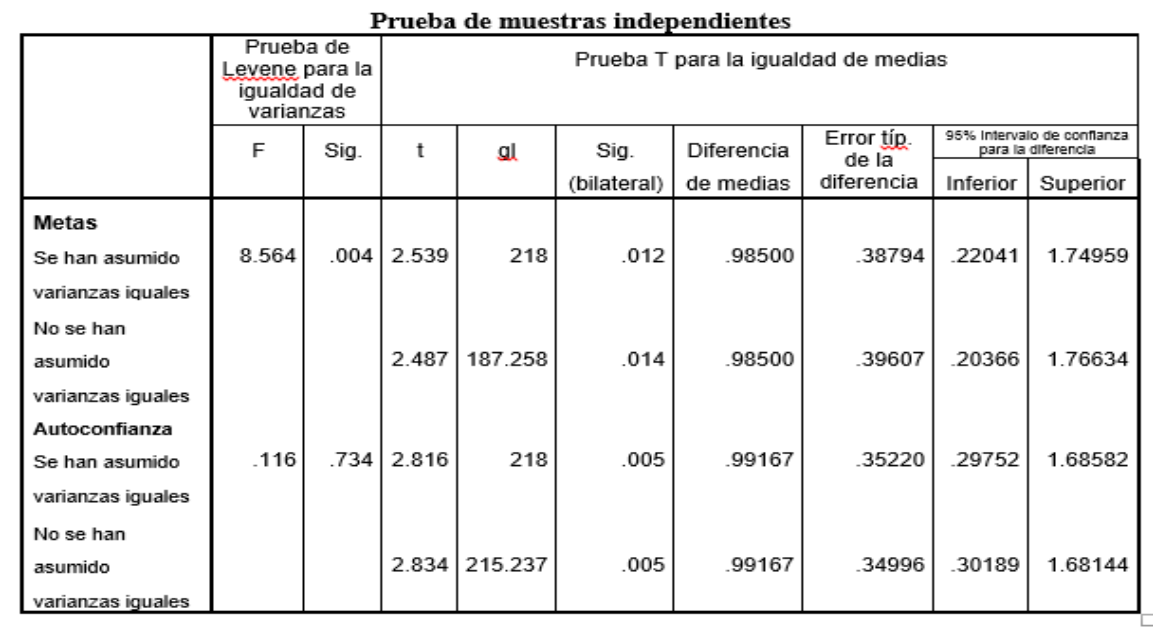

Tabla 5. Aplicación de T de Student para las características Metas y Autoconfianza 
Por último, en la Tabla 6. de las medias de las dos características México tiene un valor más alto en ambos casos.

Estadísticos de grupo

\begin{tabular}{|l|l|r|r|r|r|}
\hline & Licenciatura & \multicolumn{1}{|c|}{$\mathrm{N}$} & \multicolumn{1}{c|}{ Media } & Desviación típ. & $\begin{array}{c}\text { Error típ. de la } \\
\text { media }\end{array}$ \\
\hline \multirow{2}{*}{ Metas } & LAM & 120 & 19.7750 & 2.54526 & .23235 \\
& LAMC & 100 & 18.7900 & 3.20761 & .32076 \\
\multirow{2}{*}{ Autoconfianza } & LAM & 120 & 19.0917 & 2.68202 & .24483 \\
& LAMC & 100 & 18.1000 & 2.50050 & .25005 \\
\hline
\end{tabular}

Tabla 6. Medias de las características de Metas y Autoconfianza

\section{Diseño de formación de emprendedores}

Contar con lineamientos estratégicos en las entidades de apoyo al emprendimiento tales como las universidades, posibilita el diseño de la formación de emprendedores y también implica tomar la responsabilidad de desarrollar habilidades pertinentes. Para ello es recomendable iniciar con el autoconocimiento de talentos.

Hay que ser claros al transmitir a los estudiantes que, para crear una empresa, es decir el rol del emprendedor como empresario, además del producto o servicio, debe demostrar que conoce el mercado y que la tecnología aplicada a su proyecto resolverá un problema. De ahí que se debe resaltar la importancia de planificar actividades y contar con información necesaria para elaborar un modelo de negocios, para lo cual la práctica y uso de dinámicas es fundamental.

Sin embargo, no basta sólo con la enseñanza, sino que es importante llevar a los estudiantes a reflexionar acerca temas como la iniciativa para la búsqueda de oportunidades y la perseverancia necesaria para superar los retos que surgen en el camino. Estas características podrían ser potenciadas al participar en diálogos con emprendedores exitosos, lecturas de casos y foros.

Algunas herramientas que vendrían a potenciar las características empresariales personales son: metodología start up, fundamentos de mercadotecnia, habilidades directivas y herramientas económicas financieras.

La formación de una actitud emprendedora en los estudiantes está relacionada directamente con la innovación, por lo que requiere de un entorno favorable y un ambiente que le apoye. La universidad aún con sus limitaciones, cualesquiera que sean, para el desarrollo de características empresariales personales, tiene un papel fundamental. El rol del educador debiera posibilitar que los estudiantes sean capaces de explicar por qué suceden las cosas de determinada manera.

Darse cuenta de que las competencias asociadas a la práctica posibilitan la innovación facilitaría el desarrollo de dichas características empresariales personales. Así, el emprendedor es capaz de actuar de acuerdo con esta dinámica, asimilando la información, la integración de los conocimientos y transformando las competencias en capacidad emprendedora (Leite, 2013), como se muestra en el siguiente esquema. 


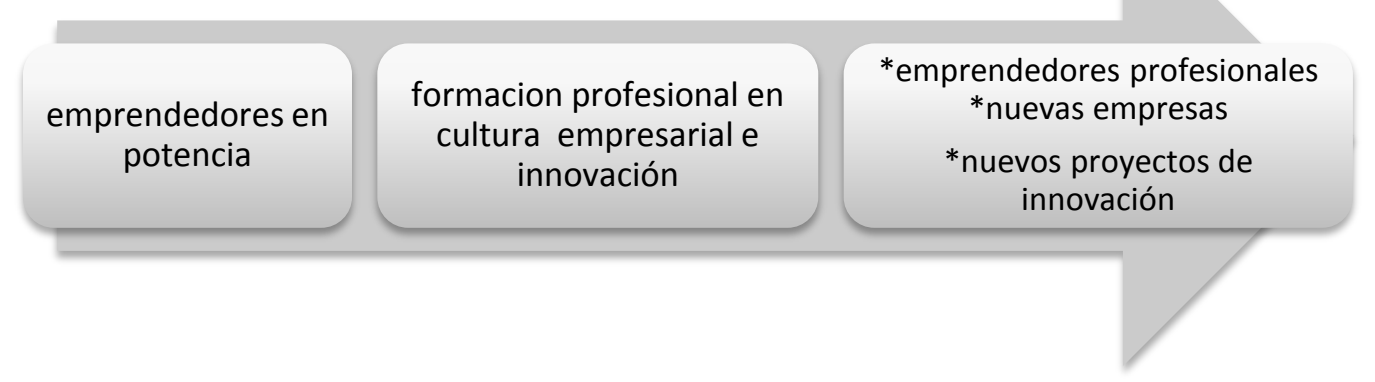

Esquema 1. Cadena Emprendimiento - Innovación. Fuente: Leite, 2013.

Finalmente, para la integración de estrategias es destacable que muchos empresarios coinciden en que, aunque la formación específica pudo ser suplida con experiencia y acceso al mercado, esto no está fácilmente al alcance de los universitarios, por lo que diseñar la formación emprendedora en universidades también implica encuentros con cámaras y asociaciones a fin de profundizar en el tema.

\section{Conclusiones y recomendaciones}

Las instituciones de educación superior están integrando con mayor frecuencia aportaciones en el tema de formación de emprendedores para mejorar los planes de estudio, de ahí que investigaciones como la presente pudieran servir de orientación para tal objetivo.

El diseño de la formación de emprendedores y el desarrollo de habilidades pertinentes no sólo es cuestión de transmitir conocimientos y enseñar herramientas, requiere del establecimiento de lineamientos estratégicos que estimulen y potencialicen las características empresariales personales.

En esta investigación se ha encontrado que existe una cantidad importante de estudios previos acerca del tema del emprendimiento que demuestran la importancia de clarificar dichas características que inducen al emprendimiento favorable. El impulso a profesionistas emprendedores debiera iniciar con una evaluación de los talentos inherentes que pueden ser medidos con alguno de los instrumentos disponibles para tal efecto.

La generación de un perfil emprendedor en los dos grupos de estudio y su posterior análisis llevan a destacar que a mayor iniciativa en la búsqueda de oportunidades para emprender mayor persistencia en las actividades relacionadas. Mientras que a mayor planificación de tareas se logra contar con mayor información para la toma de decisiones.

La comparación de resultados de los estudiantes de la licenciatura en Administración en dos universidades públicas en México y Colombia identifica ventajas y desventajas que pudieran ser aprovechadas en trabajos colaborativos.

Los resultados de esta investigación abren nuevas puertas de análisis hacia enfoques más específicos respecto al tema del emprendimiento que debieran ser abordados en futuras investigaciones.

\section{Referencias}

Alcaraz R., Rafael (2011). El emprendedor de éxito. Cuarta edición, México:Mc Graw Hill. Bygrave, W. Minniti, M. (2000). "The Social Dynamics of Entrepreneurship". Entrepreneurship: Theory and Practice. Vol. 24. Núm.3 León, España: Ed. Universidad de León, pp 25-36. 
Bremes E. y Harr J. (2016). El futuro del emprendimiento en Latinoamérica. $2^{\mathrm{a}}$ ed., México: Cengage Learning.

Campos, Figueroa y Sandoval (2014). Memorias XV Congreso Internacional sobre Innovaciones en docencia e investigación en Ciencias Económico Administrativas Zacatecas: México.www.fca.uach.mx/apcam/2014/04/.../Ponencia\%20147-UPZacatecas/recuperado $11 / 02 / 2016$

Cano, Carlos; García, Juan, y Gea, Ana. (2003). Actitudes emprendedoras y creación de empresas en los estudiantes universitarios, (146-160) Almería, España:Servicio de Publicaciones de la Universidad de Almería y Consejo Social de la Universidad de Almería

Duarte, Tito, Ruiz Tibana, Myriam (2009). Emprendimiento, una opción para el desarrollo Scientia Et Technica [en linea] 2009, XV (Diciembre-Sin mes): [Fecha de consulta: 15 de agosto de 2016] Disponible en:<http://www.redalyc.org/articulo.oa?id=84917310058> ISSN $0122-1701$

DRAE Diccionario Real Academia Española, 2016.

Formichella, M. Martha (2002). El concepto de emprendimiento y su relación con la educación, el empleo y el desarrollo local, Monografía, Buenos Aires, Argentina:Arroyos, INTA

Gibb, Allan (1999). Creating an entrepreneurial culture in support of SMEs, Small Enterprises Development" Vol.10, no.4.

Gilder, G. (1984). The Spirit of Enterprise, Nueva York: Simon \& Schuster; trad. esp. (1986): El espíritu de empresa, Madrid: Espasa- Calpe.

Global Entrepreneurship Monitor. Informe Ejecutivo, 2010 GEM:España

Global Entrepreneurship and Development Institute. https://thegedi.org/global-entrepreneurshipand-development-index/recuperado el 30 de marzo de 2016

Leite, Emanuel (2013). Empreendedorismo, Inovação, Incubação de Empresas e a Lei de Inovação - Startups . Recife. Brasil:Edições Bagaço

Páez S., Diana y García R., Johana (2011). Acercamiento a las características del universitario emprendedor en la unidad de emprendimiento empresarial de la Universidad Nacional de Colombia Revista Escuela de Administración de Negocios, () 52-69. Recuperado de http://www.redalyc.org/articulo.oa?id=20623157005

Prieto, Carlos (2014). Emprendimiento. México:Pearson.

Rodríguez, Alfonso (2009). Nuevas Perspectivas para entender el Emprendimiento Empresarial”, Pensamiento \& Gestión $\mathrm{N}^{\circ} 26.2$ Recuperado de http://rcientificas.uninorte.edu.co/index.php/pensamiento/article/view/864/4945

Schumpeter, Joseph A. (1951). Economic Theory and Entrepreneurial History. In R. V. Clemence, ed., Essays on Economic Topics of Joseph Schumpeter. Port Washington, NY: Kennikat Press.

Spinelli, Stephen. Jr. \& Adams, Robert (2012). New venture creation. Entrepreneurship for the $21^{\text {st }}$ century. 9 ed. U.S.A: Mc-Graw-Hill Irwin

UNESCO, (2015). Declaración de Incheon: Educación 2030. http://es.unesco.org/worldeducation-forum-2015/about-forum/declaracion-de-incheon

Uriarte, J., González, P. (2007). Métodos e instrumentos de evaluación psicológica de jóvenes emprendedores. España. http://www.psicologiacientifica.com/bv/psicologia-264-1-metodosenstrumentos-de-evaluacion-psicologica-de-jovenes-.html

Vargas, José. (2007). La culturocracia organizacional en México. Edición electrónica gratuita. Texto completo en www.eumed.net/libros/2007b/ 301

Veciana, José María y Urbano, D. (2000). Investigación sobre la asignatura de Creación de Empresas en la universidad española. Barcelona, España: Departamento de Economía de la Empresa (Universidad Autónoma de Barcelona). 